\title{
Three-dimensional temperature and velocity measurements in fluids using thermographic phosphor tracer particles
}

\author{
M. Stelter ${ }^{1 *}$, F. J.W.A. Martins ${ }^{1,2}$, F. Beyrau ${ }^{1}$, B. Fond ${ }^{1}$ \\ ${ }^{1}$ Institut für Strömungstechnik und Thermodynamik, Otto-von-Guericke-Universität Magdeburg, Germany \\ ${ }^{2}$ Current address: Institut für Verbrennung und Gasdynamik, Universität Duisburg-Essen, Germany \\ *moritz.stelter@ovgu.de
}

Many flows of technical and scientific interest are intrinsically three-dimensional. Extracting slices using planar measurement techniques allows only a limited view into the flow physics and can introduce ambiguities while investigating the extent of 3D regions. Nowadays, thanks to tremendous progress in the field of volumetric velocimetry, full 3D-3C velocity information can be gathered using tomographic PIV or PTV hence eliminating many of these ambiguities (Discetti and Coletti, 2018; Westerweel et al., 2013). However, for scalar quantities like temperature, 3D measurements remain challenging. Previous approaches for coupled 3D thermometry and velocimetry combined astigmatism PTV with encapsulated europium chelates particles (Massing et al., 2018) or tomographic PIV with thermochromic liquid crystals particles (Schiepel et al., 2021). Here we present a new technique based on solid thermographic phosphor tracer particles, which have been extensively used for planar fluid temperature and velocity measurements (Abram et al., 2018) and are applicable in a wide range of temperatures. The particles are seeded into a gas flow where their 3D positions are retrieved by triangulation from multiple views and their temperatures are derived from two-colour luminescence ratio imaging. In the following, the experimental setup and key processing steps are described before a demonstration of the concept in a turbulent heated jet is shown.

The measurement system is illustrated in fig. 1a. Two lasers are employed as light sources, a double-pulse green laser $(532 \mathrm{~nm})$ for particle position and velocity measurements using Mie scattered light and a singlepulse UV laser ( $266 \mathrm{~nm}$, synchronized with the first green laser pulse) to excite the phosphor luminescence. The laser beams are superimposed and shaped to illuminate a $5 \mathrm{~mm}$ diameter probe volume. A gas jet with co-flow emanating from the square tube below the laser beams is seeded with phosphor particles. To reconstruct 3D particle locations and velocities, particle images are acquired using four cameras (P1-P4) in double-frame single-exposure configuration. To maintain a large depth of field and to minimise reconstruction errors, these cameras' lenses are equipped with Scheimpflug adapters and stopped down to f/16. The emitted luminescence is captured simultaneously by two separate cameras (T1 and T2) with fully opened apertures to maximize signal levels. As a result, not all particles are imaged in focus (fig. 1b). Cameras T1 and $\mathrm{T} 2$ are equipped with specific spectral filters so that the ratio of luminescence intensities between both cameras' images is a monotonic function of temperature (fig. 1c). As described in the next paragraph, each sextet of particle images is used for extraction of particle position, velocity and temperature information.

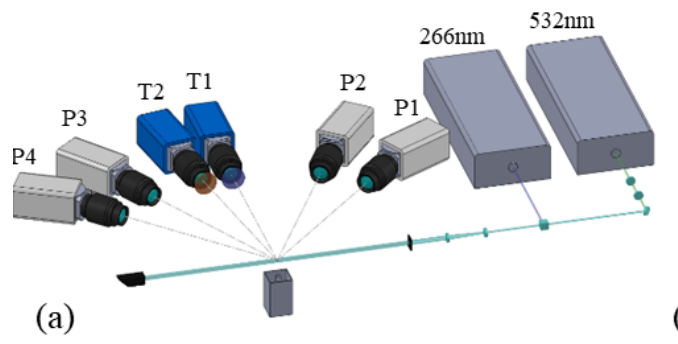

(b)
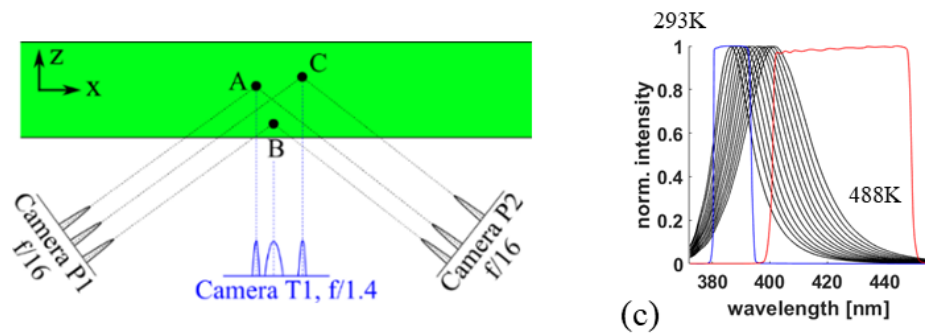

Figure 1: Experimental setup (a) with schematic of particle image formation in PTV and luminescence cameras (b) and normalized $\mathrm{ZnO}$ emission spectra from $293 \mathrm{~K}$ to $488 \mathrm{~K}$ in $15 \mathrm{~K}$ intervals showing its temperature induced shift (c). Normalised transmission profiles of the spectral filters are added in red and blue. 
First, all six camera views are calibrated by translating a 2D dot-target through the measurement volume. Then, the 3D reconstruction of particle positions in both $532 \mathrm{~nm}$-illuminated frames is performed by an inhouse particle triangulation code using the images of cameras $\mathrm{P} 1$ to $\mathrm{P} 4$. The reconstructed positions from the first frame are then projected onto the images of cameras T1 and T2. Particles are paired with these positions based on the closest particle image centre. The luminescence signal for each particle is spatially integrated within the images of cameras T1 and T2 using a 2D-Gaussian fit-based mask. A ratio is calculated from these intensities and is converted to temperature using calibration data, resulting in a discrete 3D temperature field. Particle velocity is assessed based on the displacement of paired particles between both reconstructed fields. A first displacement guess is gained by a $3 \mathrm{D}$ ensemble cross-correlation and then pairing is achieved by the closest particles.

As proof of concept, a demonstration experiment was performed using a $4 \mathrm{~mm}$ jet heated to a nozzle exit temperature of $383 \mathrm{~K}$ at a bulk velocity of $28 \mathrm{~m} / \mathrm{s}$ surrounded by a low-velocity co-flow at room temperature. Both flows were seeded with $\mathrm{ZnO}$ particles (volume equivalent diameter $600 \mathrm{~nm}$ ). 3D data of particle temperature and velocity accumulated over 366 single-shot measurements are plotted in fig. 2. As expected, hot particles are grouped within a cylindrical region corresponding to the jet flow showing an axisymmetric temperature distribution. Data from a cylindrical $2 \mathrm{~mm}$ diameter sub-volume centred in the jet flow region and considered isothermal were used to establish the particle-to-particle temperature precision, which was $18 \mathrm{~K}$ for a mean temperature of $369 \mathrm{~K}$. Highest particle velocities are also detected at the jet centre and their values decrease with radial distance. The present results demonstrate the ability of our new technique to measure 3D temperature and velocity fields. Future work will concentrate on improving the measurement density and decreasing the random temperature uncertainty by using more advanced 3D reconstruction algorithms and different thermographic phosphor materials.

(a)

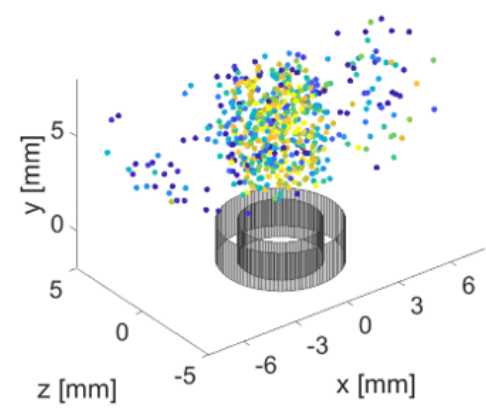

(b)

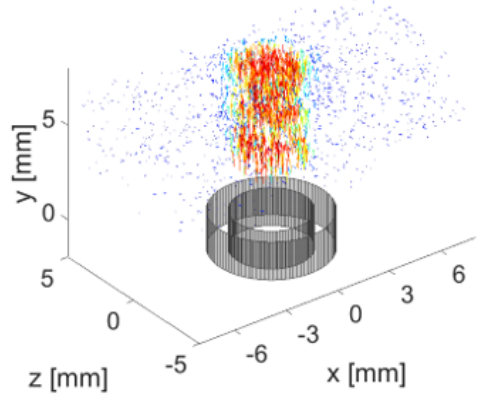

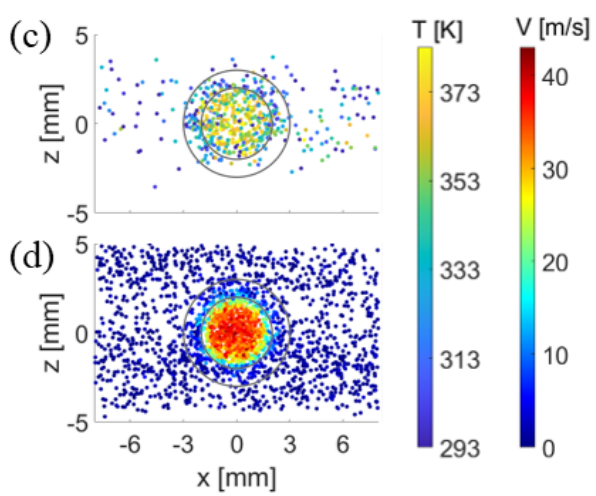

Figure 2: Reconstructed particle positions as dots with colour encoded temperature in 3D (a) and top view (c) accompanied by the particle velocity field in 3D (b) and top view (d). Inner (4 mm) and outer (6 mm) diameter of the jet nozzle are indicated in grey.

Funding from the German Research Foundation DFG (Project number 427979038) is gratefully acknowledged.

\section{References}

Abram C, Fond B, and Beyrau F (2018) Temperature measurement techniques for gas and liquid flows using thermographic phosphor tracer particles. Progress in Energy and Combustion Science 64:93-156

Discetti S and Coletti F (2018) Volumetric velocimetry for fluid flows. Meas Sci Technol 29:042001

Massing J, Kähler C, and Cierpka C (2018) A volumetric temperature and velocity measurement technique for microfluidics based on luminescence lifetime imaging. Experiments in Fluids 59:1-13

Schiepel D, Schmeling D, and Wagner C (2021) Simultaneous tomographic particle image velocimetry and thermometry of turbulent Rayleigh-Bénard convection. Meas Sci Technol 32:095201

Westerweel J, Elsinga G, and Adrian R (2013) Particle image velocimetry for complex and turbulent flows. Annual Review of Fluid Mechanics 45:409-436 